Université Michel de Montaigne Bordeaux 3

Università degli Studi di Cagliari*

\title{
SOGGETTO E VERBO CON DORMIRE, TELEFONARE E ARRIVARE IN CONTESTI DIVERSI
}

\section{INTRODUZIONE}

Quest'articolo vuole presentare lo studio realizzato su un campione di cento parlanti italiani, ed in particolare la parte che riguarda tre verbi, dormire, arrivare e telefonare, che si possono costruire con un unico argomento, il soggetto. Il punto di partenza del lavoro è la struttura argomentale identica di questi tre predicati e lo scopo è studiarne la realizzazione in due contesti enunciativi diversi: quando l'asserzione è «Predicato + Argomento» e quando la presupposizione è «Argomento» e l'asserzione è «Predicato».

I risultati del test somministrato ai parlanti ci ha portato a formulare ipotesi che riguardano la realizzazione di una stessa struttura argomentale, i rispettivi ruoli del contesto e degli elementi presenti nel determinare la strutturazione dell'enunciato ed anche le ragioni che permettono di considerare una sequenza come inadeguata o al contrario come adatta rispetto al contesto.

Inizieremo presentando la nozione di struttura argomentale e le proprietà dei tre predicati considerati. Poi introdurremo il questionario e illustreremo il suo funzionamento. Infine, presenteremo i risultati ottenuti per ogni verbo nei diversi contesti e vedremo in particolare che non ci sono due classi di verbi intransitivi ma piuttosto un continuum tra gli inergativi e gli inaccusativi.

\section{IL SOGGETTO COME ARGOMENTO UNICO}

Come abbiamo appena accennato, la base dello studio è la struttura argomentale. $\mathrm{La}$ «valenza» di un verbo - concetto introdotto da Tesnière nel 1959 - riunisce gli elementi che riguardano le entità direttamente o indirettamente coinvolte dal processo indicato dal verbo. Il punto di partenza della struttura è il verbo, che seleziona zero, uno, due, tre... argomenti necessari alla buona formazione dell'enunciato. Gli argomenti di un verbo, diversamente dai circostanti, sono supposti essere obbligatori, ma ciò non sempre è vero: si vogliono così distinguere gli argomenti necessari all'uso del predicato dagli argomenti opzionali, la cui assenza non impedisce la costruzione di un enunciato grammaticale ed interpretabile. Prendiamo l'esempio di mangiare:

\footnotetext{
* Indirizzo dell'autrice : 87, chemin de Mussonville Apt C 62, 33130 Bègles, Francia. Mél : augendre.sandra@wanadoo.fr
} 
1. a.

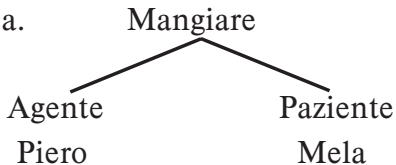

b.

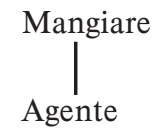

Piero

È possibile attribuire due strutture argomentali a questo predicato, una con due argomenti, un'altra con solo un agente e l'ellissi del paziente:

- Nella prima struttura, i due argomenti si distinguono per il loro ruolo semantico e per la loro funzione: «Piero» è agente e soggetto, mentre «mela» è paziente e oggetto diretto. Nel nostro esempio, sono direttamente riconoscibili per il fatto che solo il secondo può essere soggetto della frase passiva («la mela è stata mangiata da Piero» vs «??/* Piero è stato mangiato dalla mela»), ma non è sempre così. ${ }^{1}$

- Nella seconda struttura, «mangiare» si costruisce solo con un agente («Piero mangia»). Il paziente è omesso, e questo fatto conduce ad un'interpretazione che non tiene conto dell'entità che viene mangiata e che si focalizza sull'azione effettuata dal soggetto.

Questa breve presentazione della nozione di struttura argomentale pone la base dello studio empirico che proponiamo, dedicato al soggetto e alla sua realizzazione in contesto. Tale contesto è stato creato artificialmente, chiedendo ai parlanti di scegliere l'enunciato più adatto a una situazione comunicativa determinata. Si tratterà di lavorare su diverse coppie domanda-risposta. La logica, riassunta nello schema riportato sotto, è la seguente: lavorare sulla struttura argomentale di questi verbi per studiare diversi enunciati formulabili a partire da questa base, focalizzandoci sul fatto che ogni enunciato è suscettibile di ricevere diverse interpretazioni, ossia di trasmettere informazioni distinte in funzione del contesto d'enunciazione. Lo schema ha lo scopo di illustrare la complessità della situazione: con una stessa struttura argomentale, possiamo costruire diversi enunciati e ogni enunciato può anche ricevere diverse interpretazioni.

2.
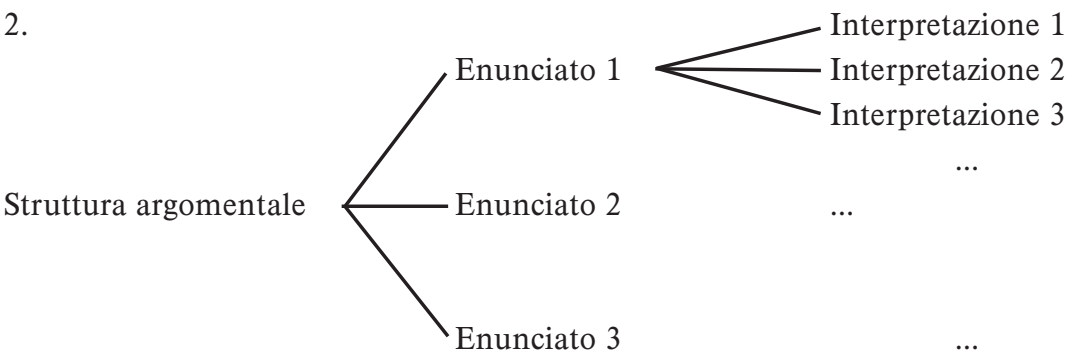

${ }^{1}$ Siccome una mela non ha la capacità di mangiare, è facile interpretarla come paziente. Invece, se il predicato mangiare avesse come argomenti Piero e squalo, i due potrebbero essere interpretati o come agenti o come pazienti. 
In modo più preciso, dopo aver preso in considerazione i tre verbi italiani suddetti, che dispongono di una stessa struttura di base (verbo + soggetto), lavoreremo a partire da un questionario somministrato a cento parlanti nativi, concentrandoci su due dei contesti che sono stati proposti loro. ${ }^{2}$

La riflessione verterà non solo sulla struttura sintattica e l'interpretazione degli enunciati, ma anche sulle differenze che esistono rispetto al contesto, al verbo selezionato e alle proprietà dell'argomento unico.

\section{DORMIRE, ARRIVARE E TELEFONARE: LE LORO CARATTERISTICHE}

Prima di tutto, vorremmo introdurre le proprietà intrinseche dei predicati considerati nell'articolo, identiche al livello della scelta dell'argomento ma diverse in termini sintattici e semantico-aspettuali.

\subsection{Proprietà comune}

Lo studio presentato in quest'articolo riguarda tre verbi italiani selezionati sulla base della seguente proprietà comune: hanno necessità di un solo argomento soggetto per la loro costruzione.

3. Dorme<smiles>C[14CH2]</smiles>

Piero

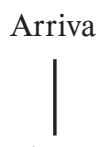

Piero

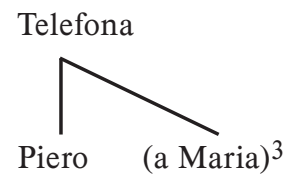

In effetti, tutti i tre verbi appartengono alla stessa classe, gli intransitivi, costruiti senza complementi e per i quali la scelta dell'ausiliare è correlata a fenomeni semantici e sintattici. Questa classe è tradizionalmente opposta a quella dei transitivi, che ammettono almeno un complemento oggetto e prendono l'ausiliare avere:

4. Verbi transitivi:

Verbi intransitivi:
SN nominativo [sv V accusativo SN...]

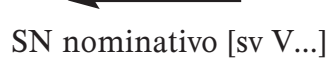

${ }^{2}$ La limitazione a due del numero di contesti studiati ha lo scopo di poter sviluppare al massimo, per ciascun contesto, le caratteristiche e i risultati e, di conseguenza, di poter fornire un contenuto il più preciso possibile nei due casi. Comunque, siamo ben coscienti che un allargamento dei dati sarebbe necessario al fine di proporre una visione globale del passaggio, in italiano, dalla struttura argomentale Predicato+Argomento all'enunciato Soggetto+Verbo.

${ }^{3}$ Consideriamo in quest'articolo solo l'uso intransitivo del verbo telefonare e non la sua possibilità di avere un paziente costruito indirettamente, perchè questo secondo argomento, al contrario del soggetto, non è necessario alla sua realizzazione. 
I tre predicati considerati richiedono esplicitamente solo un soggetto per la loro realizzazione; ci sembra pertinente introdurre l'idea che, per ciascuno dei tre verbi, è più o meno possibile riallacciare implicitamente altri elementi alla struttura argomentale:

- Dormire può essere associato ad un luogo, un modo, una durata... tanto varrebbe dire che il predicato non conduce a nessuna interpretazione specifica.

- Arrivare sottintende abbastanza direttamente un luogo («qualcuno arriva da qualche parte»), anche se il modo o il momento, per esempio, possono essere considerati come elementi pertinenti rispetto all'azione verbale.

- Quanto a telefonare, include semanticamente un paziente che riceve la chiamata, anche se non è presente nell'enunciato.

\subsection{Proprietà distintive}

Anche se possiedono una struttura argomentale identica, dormire, telefonare e arrivare non condividono le stesse caratteristiche. In effetti, la classe degli intransitivi non è omogenea e si divide in due gruppi che hanno proprietà specifiche: gli inaccusativi e gli inergativi. La distinzione tra questi due tipi di verbi si basa più che altro su delle caratteristiche sintattiche e semantiche.

\subsubsection{Livello sintattico}

Al livello sintattico, i generativisti considerano che l'argomento unico degli inergativi è soggetto a tutti i livelli di rappresentazione (stesse proprietà sintattiche del soggetto dei verbi transitivi), invece quello degli inaccusativi è un oggetto (struttura profonda) o un soggetto (in superficie). Questo elemento viene allora considerato da questi teorici come esterno al SV per un verbo inergativo, e interno al SV per un inaccusativo:

5.

- Verbo inergativo: NP [VP V]

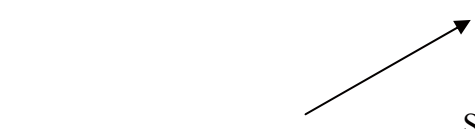

Struttura intransitiva:
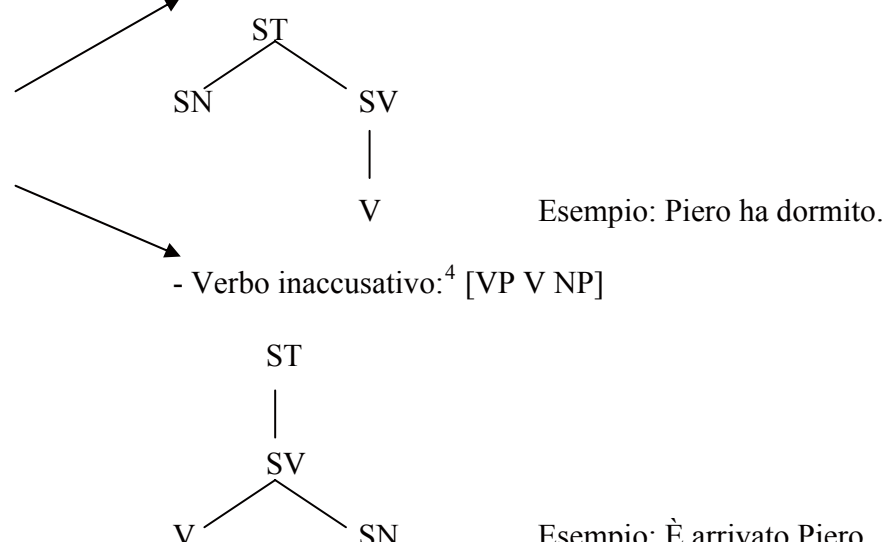

Esempio: È arrivato Piero.

\footnotetext{
${ }^{4}$ Burzio (1986) parla di verbi «ergativi» rispetto al fenomeno analogo delle lingue dette «ergative» nelle quali il soggetto dell' intransitivo ha lo stesso caso dell' oggetto diretto del transitivo.
} 
L'ipotesi secondo la quale il soggetto di un verbo inaccusativo occupa la posizione vuota dell'oggetto è condivisa da molti linguisti, che suppongono un pro (locativo / temporale per Pinto 1997) prima del verbo, che permette di avere l'argomento unico in posizione postverbale. Tuttavia, non siamo convinti della distinzione di due posizioni sintattiche per un soggetto postverbale a seconda che il verbo sia inaccusativo o no. In effetti, da un lato, l'argomento unico degli intransitivi rimane un soggetto sintattico qualunque sia il verbo e, dall'altro, la posizione che occupa nell' enunciato può essere studiata solo come una scelta del parlante rispetto al suo bisogno comunicativo e non come una necessità sintattica poichè, da questo punto di vista, la sua posizione in italiano è libera.

In una lingua come il francese, l'argomento spesso usato per distinguere dagli altri i verbi inaccusativi è che solo questi possono iniziare una frase, anche se è possibile trovare qualche contro-esempio, come quello del verbo non-inaccusativo adopter/adottare che può essere messo all'inizio, come in «Adoptent ce point de vue tous les membres du conseil / Adottano questo punto di vista tutti i membri del consiglio». L'italiano, che dispone dell'inversione libera, può mettere qualsiasi verbo all'inizio. Di conseguenza, in italiano questa possibilità sintattica non è pertinente per identificare i verbi inaccusativi. In italiano infatti, per poter definire la classe dei verbi inaccusativi sono utilizzati diversi criteri. Tra l'altro la scelta dell'ausiliare essere, la possibilità di avere un soggetto con il participio assoluto del verbo, il test del ne partitivo o genitivo ${ }^{5}$ (cf. Andorno 2003: 105 o Benincà 1994: 69-72, per maggiori dettagli) o ancora il fatto che solo i verbi inergativi possono formare un nome d'agente con il suffisso -ore (cf. Graffi 1994: 57 per quest'ultimo argomento)... Appartengono a questa categoria gli intransitivi che richiedono l'ausiliare essere, i verbi ergativi, i verbi riflessivi ed ogni verbo usato nella forma passiva.

Questa distinzione tra verbi non inaccusativi e inaccusativi viene messa in relazione diretta con lo statuto del soggetto nei due casi: l'argomento unico di un non inaccusativo possiede tutte le caratteristiche proprie a questo tipo di costituente, invece quello di un verbo inaccusativo riceve anche alcuni tratti dell'oggetto. Di conseguenza, dal punto di vista posizionale, l'ordine SV viene tradizionalmente associato ai verbi del primo tipo mentre l'ordine VS è considerato come più naturale rispetto all'ordine SV con gli inaccusativi. ${ }^{6}$ Adottano quest'idea Frison (1988) e Cornish (2001), tra l'altro,

\footnotetext{
${ }^{5}$ Questo argomento evidenzia il fatto che ne può solo corrispondere ad un complemento del nome proveniente dal COD di un verbo transitivo o al soggetto di un verbo inaccusativo. Ma anche in questo caso, un verbo come telefonare pone problemi. La questione è, secondo gli autori, se considerare come grammaticale un enunciato come "ne hanno telefonato tre», o se considerare (Frison 1988: 50) il ne partitivo accettabile, ma solo senza ausiliare, oppure, sia con ausiliare che senza, considerare agrammaticale l'enunciato (Burzio 1986: 22; Graffi 1994: 57).

6 Oltre al tipo di verbo, altri fattori (sintattici, semantici, comunicativi...) possono spiegare la scelta del parlante tra SV et VS, come il tipo di proposizione o la presenza di un altro complemento all'inizio dell'enunciato. Per maggiori dettagli su quest'insieme di criteri rinviamo allo studio di Sasse (1995) e ai lavori su corpus di Sornicola (1995) o Bernini (1995), tutti e tre reperibili in Matras/Sasse (1995).
} 
che considerano che questi verbi hanno naturalmente il loro soggetto in posizione postverbale, come quella dell'oggetto, o anche Marandin (2003) che parla di «costruzione inaccusativa» e non di inversione del soggetto. Al contrario, altri linguisti parlano di spostamento del soggetto (Costabile 1967) o notano semplicemente questa preferenza per la postposizione con queste costruzioni (Genot 1989: 30 e seguenti ${ }^{7}$ ).

Qui non mettiamo in discussione le proprietà sintattiche che permettono di distinguere due tipi di verbi mono-argomentali ma tenteremo, a partire dalle scelte dei parlanti nel test, di mostrare che la relazione verbo-soggetto in un contesto e che la costruzione del soggetto e la sua interpretazione oltrepassano la dicotomia verbi in accusativi - verbi non inaccusativi.

\subsubsection{Livello semantico}

La distinzione tra i due tipi di verbi intransitivi può anche essere considerata come semantica, determinata dall'interazione di parametri come l'aspetto, o il ruolo semantico del soggetto: un verbo inergativo denota piuttosto un'attività incompiuta (atelica), un verbo inaccusativo piuttosto uno stato resultativo (telico) e al livello del ruolo semantico dell'argomento unico, è piuttosto agentivo se il verbo è inergativo, mentre è non agentivo se è inaccusativo. Questi criteri, che permetterebbero di distinguere i verbi inaccusativi dai verbi inergativi, non ci sembrano né affidabili né sufficienti per dividere in due la classe dei verbi intransitivi. Infatti, in italiano, le caratteristiche aspettuali e semantiche dei verbi non permettono di predire il loro comportamento sintattico. In effetti, se si considerano i tre verbi di quest'articolo:

- l'agentività di un soggetto umano è innegabile per arrivare (il criterio non è dunque sempre affidabile);

- telefonare ha dei punti in comune con i due tipi di verbi (cf. frecce; non ci sono due classi ben distinte).

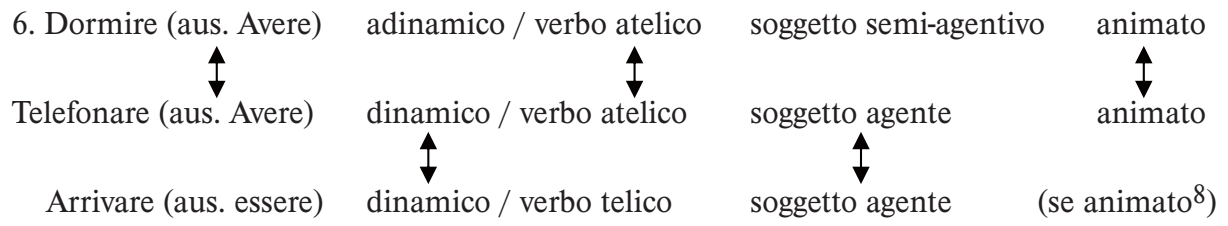

Nella stessa logica di Cennamo (2001: 431) che parla di «gerarchia» o di Loporcaro (2001: 463) che difende l'idea di una "scala», consideriamo che esiste un conti-

\footnotetext{
${ }^{7}$ Nella classifica dei diversi tipi di frasi che propone, sottolinea che pone il soggetto dei verbi inaccusativi alla destra del verbo solo perché «queste frasi ammettono spesso l'ordine V SN non marcato» (1989: 30).

8 Con un enunciato come «la lettera arriva», bisogna dire che l'agentività e l'animatezza non sono sempre propriétà che permettono l'identificazione dell'argomento unico di questo verbo.
} 
nuum tra la classe degli inaccusativi e quella degli inergativi: si tratta di considerare che tra un verbo inergativo come dormire e un verbo inaccusativo come arrivare, possono essere classificati come intermedi verbi del tipo telefonare. Per quanto riguarda la scelta dell'ausiliare e l'aspetto atelico dell'azione, telefonare si avvicina a dormire, ma in termini di dinamismo dell'azione e di ruolo semantico del soggetto è simile ad arrivare.

\section{COSTRUZIONE ED INTERPRETAZIONE DEL SOGGETTO: DATI ED ANALISI}

Dopo aver presentato le nozioni di base di questo studio, stiamo per introdurre il questionario e l'analisi dei risultati. Si precisa subito che questo lavoro non è uno studio su corpus, come per esempio quelli di Sornicola (1995) o Bernini (1995), ma è proprio una ricerca su parlanti nativi, con lo scopo di partire da un contesto preciso per arrivare all'insieme degli enunciati che gli corrispondono.

\subsection{Presentazione del questionario}

I dati presentati più avanti sotto forma di grafici sono stati raccolti a partire da un questionario distribuito a Cagliari (Sardegna) a cento parlanti nativi ${ }^{9}$ per l'elaborazione della nostra tesi in cotutela tra le università di Bordeaux e di Cagliari. In Sardegna, come dappertutto in Italia, l'italiano è marcato in diatopia. Di conseguenza, fare un'indagine sul parlato cagliaritano o di qualsiasi altra regione ci sembra essere ugualmente rilevante, soprattutto perché lo scopo del lavoro non è quello di paragonare i risultati del test su base regionale. La tesi, e quindi il questionario complessivo, propone lo studio della realizzazione dei due argomenti diretti (il soggetto e l'oggetto diretto). Per questa ragione, anche se studieremo in quest'articolo solo il caso di dormire, telefonare e arrivare, l'intero test si divide in cinque parti, ognuna dedicata ad un particolare verbo. ${ }^{10}$

Il lavoro procede nel modo seguente: per ogni verbo sono proposte diverse domande o enunciati di base (pronunciati da un parlante A) ed a ognuno di questi contesti viene associato un insieme di enunciati di risposta (B1, B2, B3...) sui quali la persona interrogata deve riflettere (parlante $\mathrm{B}$ ), scegliendo quelli più adatti all'input dato da $\mathrm{A}$. Le risposte proposte contengono sempre almeno il verbo studiato e/o il suo soggetto:

- In un primo tempo, viene chiesto di mettere tra parentesi o di barrare le forme considerate come non adatte (cioè marcate $o$ inadeguate rispetto al contesto).

\footnotetext{
${ }^{9}$ Le persone interrogate, 42 uomini e 58 donne, hanno 15-20 anni e 66-70 anni, sono nati a Cagliari (66) e risiedono (78), per la maggior parte a Cagliari città o nella periferia. Quanto a grado di istruzione, 44 sono laureati mentre 56 hanno un livello di studio inferiore e, per quel che riguarda il loro lavoro o stato, i dati rappresentani settori abbastanza vari (commercianti, studenti, dottorandi, impiegati, insegnanti, educatori, disoccupati...).

${ }^{10}$ I cinque verbi considerati nel questionario complessivo sono i seguenti: dormire, arrivare, telefonare, mangiare e rievocare. Oltre ai tre verbi monoargomentali considerati nell'articolo, ci sono anche due verbi transitivi, usati al fine di studiare la realizzazione dell'oggetto diretto.
} 
- In un secondo tempo, si chiede di classificare gli enunciati ritenuti pertinenti in ordine di preferenza, scrivendo dei numeri nelle caselle corrispondenti (1 in quella situata davanti all'enunciato preferito, 2 in quella situata davanti al secondo preferito...).

- Poi, in un terzo tempo, i parlanti possono scrivere altre forme di risposta che considerano come adatte al contesto proposto, che contengano comunque il verbo e/o il soggetto studiato.

È stato anche precisato che:

- quanto è posto tra parentesi nelle domande o nelle risposte deve essere considerato come facoltativo;

- le maiuscole sono utilizzate per indicare l'accentuazione;

- la virgola denota una pausa.

Consideriamo ora un esempio preso dal test:

\section{A: Ha dormito Piero? / È Piero che ha dormito? $\longleftarrow$ Contesto linguistico imposto (Parlante fittivo A)}

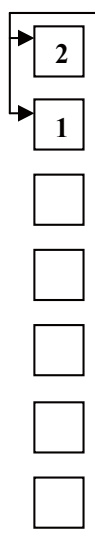

B1: No. Ha dormito MARIA.

Esempio di enunciati scelti

B1: No. Ha doImito MARIA.

B2: No. MARIA ha DORMITO.

B3: No. È MARIA che ha DORMITO.

B4: No. (È) MARIA.

(B2 in la scelta e B1 in 2a)

B5: No. Ha DORMITO, Maria.

B6: No. MARIA, ha DORMITO.

B7: No. C'è MARIA che ha DORMITO.

Esempio di enunciato escluso

Altre risposte possibili, con costruzioni diverse: Aggiunta di altri enunciati adatti

Come abbiamo già sottolineato, ci limiteremo in questo articolo a presentare due casi che riguardano i verbi dormire, arrivare e telefonare:

1) - «Che cosa è successo?»

2) - «Sono andata a fare spese. E Maria?» 
Tuttavia, vogliamo precisare che l'insieme del lavoro contiene un numero molto più ampio di contesti («Chi+verbo?», «che cosa hai fatto stamattina?», «ha/è+verbo+qualcuno?», «ha/è+verbo+Piero?» $\left.{ }^{11} \ldots\right)$.

\subsection{Dati e analisi}

Abbiamo scelto di presentare i risultati ottenuti a partire dai cento questionari avvalendoci di due grafici per ogni contesto:

- Il primo è un istogramma che descrive le quattro o cinque prime scelte (a seconda dei contesti) dei parlanti e anche i tassi di esclusione. Suo scopo è seguire un enunciato attraverso le decisioni che lo riguardano, ma anche dare una visione d'insieme sull'esclusione di tutti gli enunciati della lista.

- Il secondo, più generale, presenta il totale dei punti ottenuti ${ }^{12}$ dalle diverse proposizioni di risposta, sotto la forma di percentuali. Ogni settore del grafico corrisponde a un enunciato, e ciò permette di valutare il peso di ognuno rispetto all'uso globale e di poter paragonare i risultati tra di loro.

\subsubsection{Primo contesto: «Che cosa è successo?»}

Il primo contesto linguistico che abbiamo scelto di studiare nel nostro studio possiede le caratteristiche seguenti:

- La domanda sottintende che qualcosa è successo.

- La richiesta d'informazione riguarda questo evento.

- La domanda non verte né su un'azione, né su un'entità/persona particolare. Di conseguenza, né il verbo studiato né il suo soggetto sono presenti in quest'ultima.

- Il parlante A suppone che B conosca la risposta, cioè che quest'ultimo sappia ciò che effettivamente è successo.

- L'interesse dell'intervento di B è quindi di rispondere alle attese di A introducendo i dettagli dell'evento.

${ }^{11}$ In questo ultimo caso, il soggetto presente nella domanda non corrisponde a quello che c'è nella risposta. In effetti, si tratta di legare l'azione espressa dal verbo con una persona diversa: il soggetto è focus contrastivo nella risposta, cioè costituisce non solo l'informazione pertinente in legame con il predicato, ma ha anche un valore oppositivo o contrastivo rettificando una identificazione sbagliata della persona associata all'azione verbale. Prendiamo un esempio semplice per illustrare questo punto:

Ha dormito Piero? / È Piero che ha dormito? (La domanda conduce al legame dormire-Piero). No. (È) Maria (che ha dormito). (La risposta rifiuta dormire-Piero ed introduce dormire-Maria).

12 L'attribuzione dei punti ai diversi enunciati viene fatta nel modo seguente: il punto di partenza è la gerarchizzazione degli enunciati $\left(1^{\circ}, 2^{\circ}, 3^{\circ}, 4^{\circ} \ldots\right)$ operata dall'insieme dei parlanti. Se, per un certo contesto, i parlanti hanno classificato fino a cinque enunciati, abbiamo attribuito cinque punti agli enunciati classificati primi, quattro punti agli enunciati classificati secondi, tre punti agli enunciati classificati terzi, due punti agli enunciati classificati quarti, un punto agli enunciati classificati quinti e nessun punto per quelli che sono stati esclusi. 
A partire da queste proprietà contestuali, consideriamo che, nella risposta, il soggetto e il verbo, o piuttosto l'azione verbale associata al referente del soggetto, formano un insieme introdotto per la prima volta nell'interazione A-B. Il contesto conduce in effetti a formulare un enunciato di tipo tetico. Dal punto di vista filosofico adottato da Sasse (1987), gli enunciati si distinguono in tetici e categorici sulla base dell'assenza (tetici) o della presenza (categorici) di una predicazione. In altre parole, secondo Sasse, in un enunciato tetico non si attribuisce una proprietà al soggetto. Dal punto di vista comunicativo adottato in questo lavoro, un enunciato tetico non è un enunciato nel quale non c'è nessuna predicazione ma un enunciato nel quale tutti gli elementi rinviano a referenti e concetti «nuovi» rispetto al contesto enunciativo. In risposta alla domanda «che cosa è successo?», l'enunciato-risposta è quindi tetico, tutti i concetti evocati dai suoi costituenti non essendo già stati attivati nella conversazione in corso. Tuttavia, anche se il soggetto e il verbo hanno qua lo stesso statuto comunicativo, non condividono le stesse proprietà nell'enunciato (posizione, accentuazione, distaccamento o no...) ed è proprio questo che dà la sua pertinenza al contesto. In effetti, i dati contestuali conducono ad un enunciato tetico attribuendo la stessa prominenza al soggetto e al verbo, ma la sintassi e la prosodia gerarchizzano in un certo senso questi due elementi dando loro delle proprietà distinte.

\section{A) Dormire}

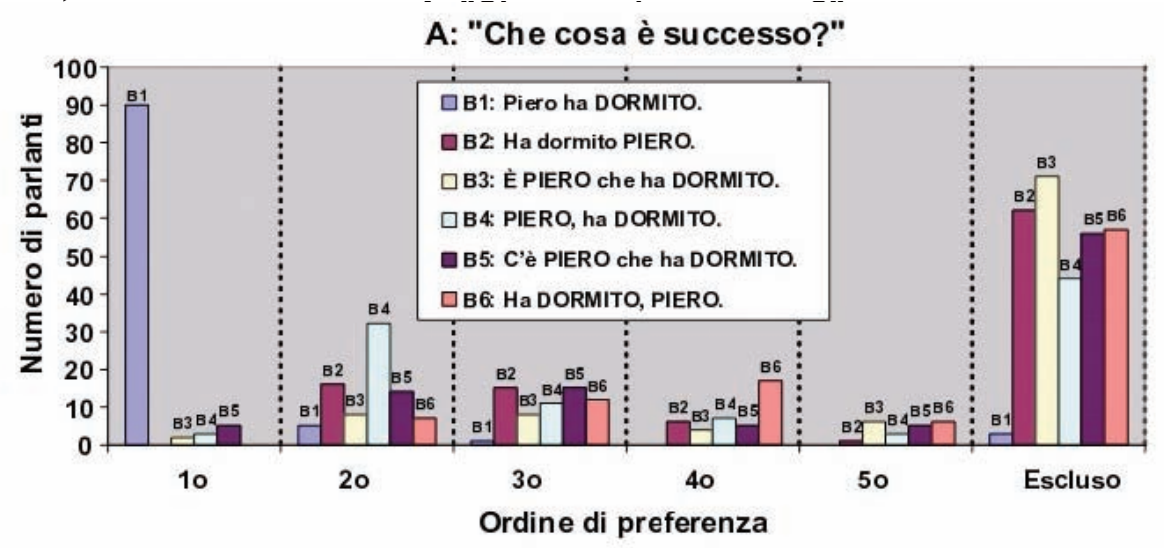

Totale dei punti per enunciato (percentuale)

A: "Che cosa è successo?"

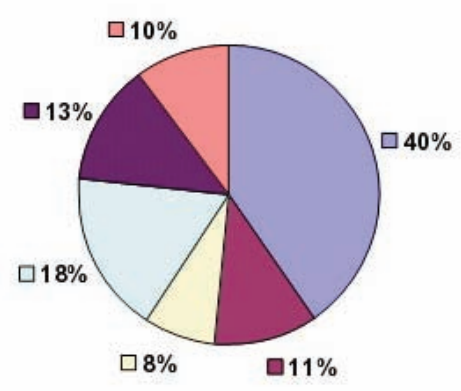

口B1: Piero ha DORMITO.

口B2: Ha dormito PIERO.

口B3: E PIERO che ha DORMITO.

口B4: PIERO, ha DORMITO.

— B5: C'è PIERO che ha DORMITO.

口B6: Ha DORMITO, PIERO. 
Da questi due primi grafici che riguardano dormire deriva la gerarchizzazione seguente, della quale dettagliamo in seguito i principali tratti:

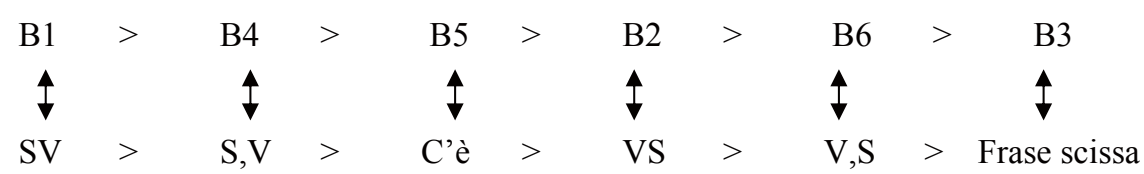

La sequenza SV (B1) è senza dubbio quella che corrisponde meglio al contesto proposto (cf. prima scelta dell'istogramma precedente). Il totale dei punti ottenuti dà una percentuale di $40 \%$, molto alta rispetto a quella dell'enunciato $\mathrm{S}, \mathrm{V}$ classificato secondo; ciò mostra la sua supremazia come risposta ad una domanda a focalizzazione larga con il verbo dormire. Questa variante consiste in un blocco unico di informazione il cui punto di partenza è il soggetto ed è il verbo a occupare la posizione finale, a portare l'accento principale e a costituire l'esito della presa di parola. ${ }^{13}$

La variante $S, V(B 4)$, che presenta la stessa disposizione degli elementi di B1 ma con un distacco a sinistra del soggetto viene classificata seconda, con una percentuale molto meno importante ( $40 \%$ per B1 contro $18 \%$ per B4). In B4, l'ordine $\mathrm{S}+\mathrm{V}$ è mantenuto, invece il blocco unico d'informazione è diviso in due: il parlante informa dell'esistenza di Piero (elemento dislocato) e poi stabilisce il legame tra questa persona e il fatto di dormire. Non studieremo in quest'articolo tutte le proprietà della dislocazione a sinistra ma rinviamo a Milano (2005) che ha effettuato un lavoro molto interessante su questo costrutto nell'italiano parlato.

Il c'è presentativo (B5), in terza posizione, è anche percepito come adatto a questo tipo di contesto (13\%). Nella stessa logica di B4 (S,V), l'apporto informativo si divide in due parti: si presenta prima il soggetto tra c'è e che e poi gli si attribuisce la proprietà di avere dormito.

L'ordine VS (B2), la dislocazione a destra del soggetto (B6) ${ }^{14}$ e la frase scissa (B3), occupano le tre ultime posizione della classifica per dormire, ottenendo le percentuali le più basse (nell'ordine: $11 \%, 10 \%$ e $8 \%$ ).

13 Segnaliamo semplicemente che le due altre possibilità di risposta che sono state proposte dai parlanti hanno questo ordine SV: si tratta di «Maria si è addormentata», aggiunto due volte, nel quale dormire viene sostituito dal suo omologo telico e di «è successo che Maria ha dormito», che riprende in parte la domanda e introduce la sequenza SV in una subordinata.

14 Come Bonvino (2005) o Sasse (1995), tra l'altro, facciamo la distinzione tra soggetto postverbale e soggetto dislocato a destra, dal punto di vista sintattico e pragmatico. 


\section{B) Telefonare}

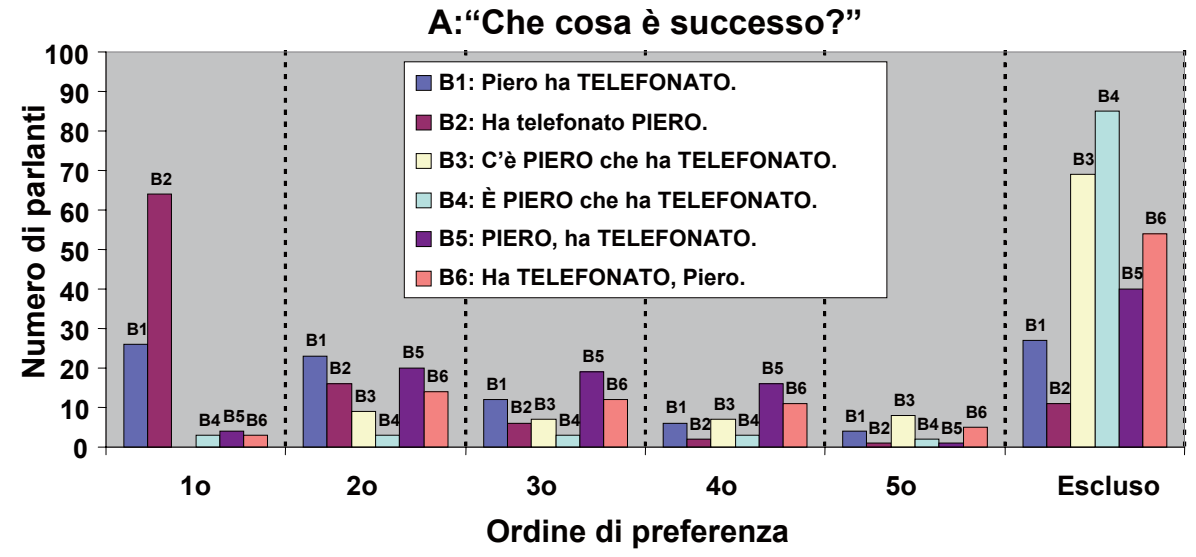

Totale dei punti per enunciato (percentuale)

A: "Che cosa è successo?"

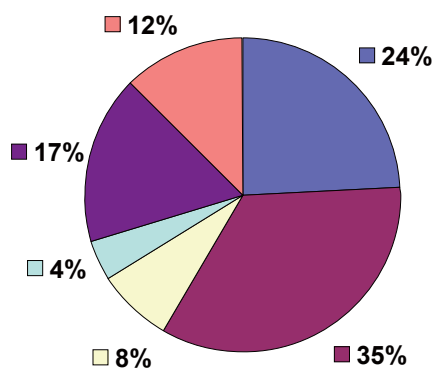

$\square$ B1: Piero ha TELEFONATO.
$\square$ B2: Ha telefonato PIERO.
$\square$ B3: C'è PIERO che ha TELEFONATO.
$\square$ B4: È PIERO che ha TELEFONATO.
$\square$ B5: PIERO, ha TELEFONATO.
$\square$ B6: Ha TELEFONATO, Piero.

Per lo stesso contesto, i risultati che riguardano il verbo telefonare sono abbastanza diversi. La gerarchizzazione degli enunciati è la seguente:

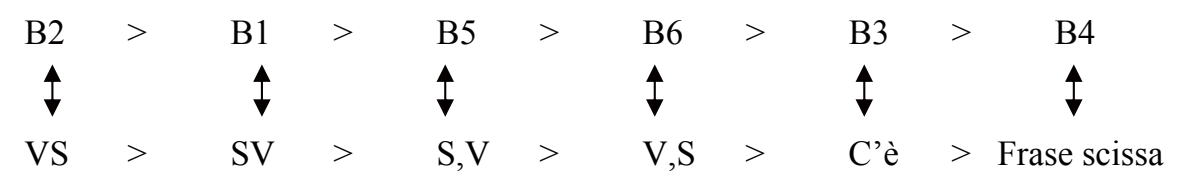

Come per dormire, approfondiamo l'analisi sui dati ed in particolare sulle tre prime scelte della classifica.

La proposizione di risposta VS15 (B2) ottiene per il verbo telefonare la prima posizione, davanti alla variante SV (35\% per B2 contro $24 \%$ per B1). Per questo verbo ed

15 Un'unica altra forma di risposta, che ravviciniamo alla proposizione VS, è stata proposta: si tratta di «è successo che ha telefonato Piero», che riprende in parte la domanda e poi introduce la sequenza VS in una subordinata. 
in questo contesto specifico, i parlanti considerano come canonico (cioè come il più adatto) un enunciato senza rottura, con il soggetto in posizione postverbale e più fortemente accentuato del verbo (cf. istogramma, prima scelta). Ciò dimostra che quando si introduce la relazione Piero/Soggetto-Telefonare/Verbo, il parlante focalizza più naturalmente il soggetto che il verbo (a differenza che per dormire).

La forma SV (B1), perfettamente opposta a VS (B2) in termini di posizioni e di accentuazioni, risulta in seconda posizione. Per il verbo considerato ed in risposta ad una domanda a focalizzazione larga, i parlanti preferiscono avere il soggetto nella posizione detta «focale» (B2) piuttosto che il verbo (B1).

$\mathrm{S}, \mathrm{V}$ (B5), come nel caso di dormire, è l'enunciato direttamente classificato dopo SV. Osservando l'istogramma, si vede chiaramente che è in terza e quarta posizione che la variante $\mathrm{S}, \mathrm{V}$ si vede attribuire i tassi i più elevati, dietro VS e SV, le forme meno complesse dal punto di vista strutturale.

L'enunciato V,S (B6), il c'è presentativo (B3) e la frase scissa (B4) sono ultimi nella classifica, con una percentuale, nell'ordine di $12 \%, 8 \%$ e $4 \%$.

\section{C) Arrivare}

\section{A: "Che cosa è successo?"}

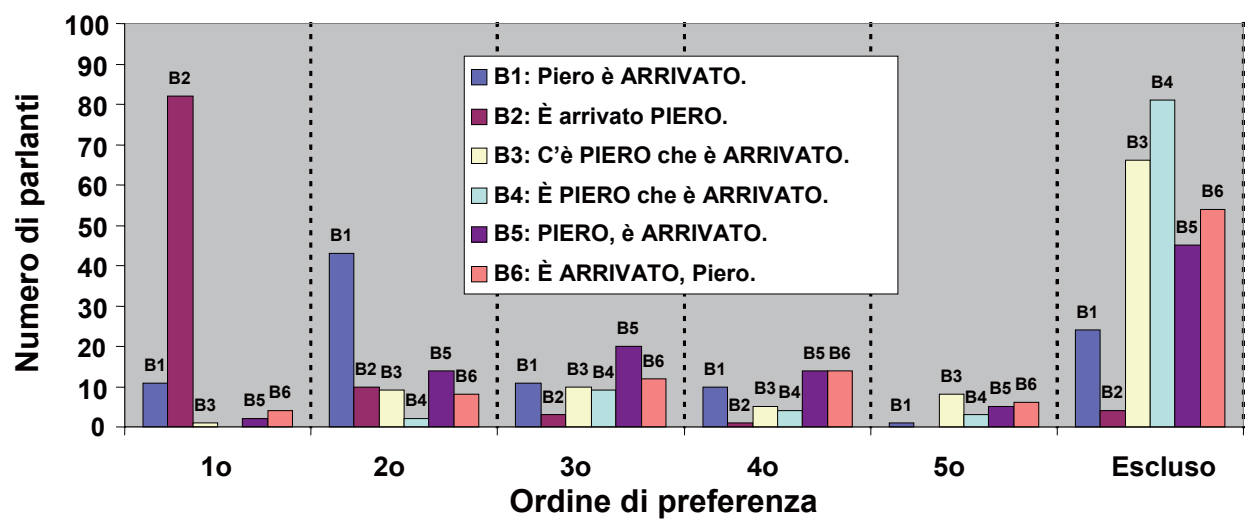

Totale dei punti per enunciato (percentuale)

A: "Che cosa è successo?"

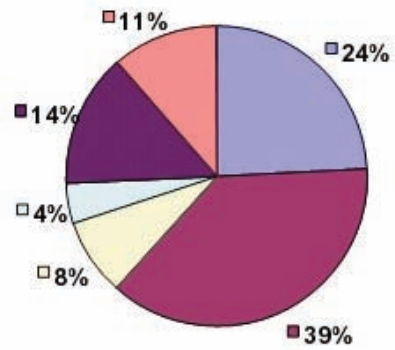

口B1: Piero è ARRIVATO.

口B2 : É arrivato PIERO.

口B3 : C'è PIERO che è ARRIVATO.

口B4 : E PIERO che è ARRIVATO.

口55 : PIERO, è ARRIVATO.

口B6: É ARRIVATO, Piero. 
Per quello che riguarda il verbo inaccusativo arrivare, i risultati sono assolutamente identici a quelli di telefonare in termini di classifica:

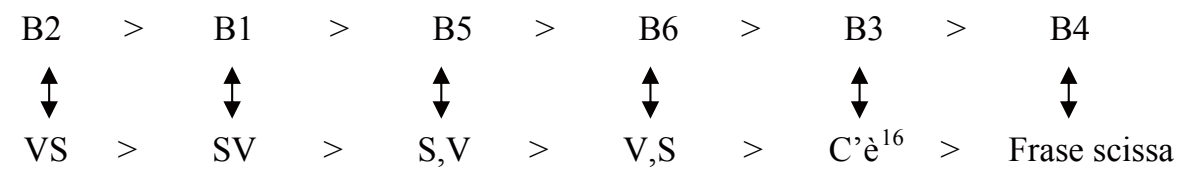

Tuttavia si deve notare che VS viene scelto come enunciato canonico da 82 parlanti su cento, invece SV solo da una decina di persone (cf. istogramma, prima scelta). Nel caso di arrivare, è molto più naturale per un parlante nativo rispondere con una sequenza VS ad una domanda a focalizzazione larga, cosa che è un po' meno evidente con il verbo precedente.

\section{D) Tabella riassuntiva e conclusione}

Dopo aver visto in dettaglio i risultati che riguardano ognuno dei tre verbi presi in considerazione, concluderemo questo primo punto con una sintesi globale e la relativa analisi:

- Sulla tendenza delle due prime scelte dei parlanti:

$\begin{array}{lll}\text { Verbi } & \text { Prima posizione } & \text { Seconda posizione } \\ \text { DORMIRE } & 90 \% \text { per SV } & 32 \% \text { per S,V } \\ \text { TELEFONARE } & 64 \% \text { per VS } & 23 \% \text { per SV } \\ \text { ARRIVARE } & 82 \% \text { per VS } & 43 \% \text { per SV }\end{array}$

- Ecco in sintesi le percentuali ottenute per ogni enunciato:

\begin{tabular}{|c|c|c|c|}
\hline Enunciati-risposte & DORMIRE & TELEFONARE & ARRIVARE \\
\hline SV & $40 \%$ & $24 \%$ & $24 \%$ \\
\hline VS & $11 \%$ & $35 \%$ & $39 \%$ \\
\hline $\mathrm{S}, \mathrm{V}$ & $18 \%$ & $17 \%$ & $14 \%$ \\
\hline $\mathrm{V}, \mathrm{S}$ & $10 \%$ & $12 \%$ & $11 \%$ \\
\hline C'è presentativo & $13 \%$ & $8 \%$ & $8 \%$ \\
\hline Frase scissa & $8 \%$ & $4 \%$ & $4 \%$ \\
\hline
\end{tabular}

(In grassetto la percentuale più alta per ogni verbo.)

${ }^{16}$ L'unica altra possibilità di risposta proposta da un parlante si avvicina al c'è presentativo: «C'è Piero. È arrivato ora». In effetti, si divide in due frasi distinte, la prima introduce la presenza di Piero e la seconda stabilisce il legame tra questa presenza con il fatto che sia arrivato (ora). 
I dati così raggruppati ci conducono, prima in termini di strutture e poi in termini di verbi, alle seguenti conclusioni:

La frase scissa non viene considerata come adatta al contesto proposto qualunque sia il verbo. Ciò può spiegarsi da un punto di vista sintattico e pragmatico: l'enunciato presenta una sintassi complessa (due blocchi con una subordinata) e la frase scissa presuppone che qualcuno «ha dormito / ha telefonato / è arrivato» e afferma che questa persona è «Piero»; invece niente nel contesto indica una tale informazione. Infatti, questa struttura deve condividere con il contesto l'implicazione espressa nella subordinata, ma non è questo il caso, da cui il suo non adeguamento.

Il c'è presentativo, che ha la funzione di introdurre nuovi elementi e azioni, viene considerato poco naturale in questo contesto per i verbi dinamici telefonare e arrivare (percentuali d'uso inferiori a $10 \%$ rispetto all'insieme di proposte), invece con dormire, adinamico, questa struttura è classificata proprio dopo gli enunciati Soggetto + verbo con o senza dislocazione del soggetto (SV e $\mathrm{S}, \mathrm{V}$ ) ma soprattutto prima della variante VS. E quest'ultimo punto ( $c$ 'è presentativo > VS) sostiene la pertinenza debole attribuita dai parlanti nativi ad un enunciato di tipo VS con dormire in un contesto in cui vengono introdotti e messi in relazione per la prima volta un referente e un'azione. Qualunque sia il verbo però, le percentuali di uso del c'è presentativo sono solo del 10\% circa, debolezza che attribuiamo alla complessità sintattica e alla pesantezza di una tale struttura.

La struttura V,S, che permette una focalizzazione dell'azione verbale associata ad una totale defocalizzazione dell'elemento distaccato, non appare per nessuno dei verbi tra gli enunciati più adatti. Questo risultato ci sembra completamente logico, nella misura in cui il soggetto (come il verbo) è qua contestualmente prominente, e deve, di conseguenza, essere marcato come tale nell'enunciato. Invece, $\mathrm{V}, \mathrm{S}$ equivale ad introdurre l'azione verbale e ad attribuirla ad un soggetto nullo in italiano («ha dormito (, Piero) / ha telefonato (, Piero) / è arrivato (, Piero)») ${ }^{17}$

Per le sequenze SV e S,V, ci sembra pertinente fonderle in una variante unica $\mathrm{S}(,) \mathrm{V}$, nella quale il soggetto è distaccato o no. In effetti, qualunque sia il verbo, i parlanti hanno fatto seguire le due strutture nella classifica e, anche se la loro scelta si è orientata prima per semplicità sull'enunciato SV, le due forme sono legate per l'ordine degli elementi che propongono e per le loro proprietà comunicative.

L'ultimo tipo di enunciato,VS, non ha lo stesso statuto per i due verbi dinamici e per l'adinamico: gli si preferisce una struttura complessa, il c'è presentativo, con dormire. Invece, gli altri due verbi hanno la proprietà comune di vedere questo enunciato (con VS) scelto dai parlanti come quello non marcato rispetto al contesto.

In termini di verbi, la situazione per il verbo non-inaccusativo dormire è la più netta: è l'ordine $\mathrm{S}+\mathrm{V}$ che prevale, prima senza dislocazione del soggetto (prima

${ }^{17}$ In una lingua a soggetto non nullo come il francese, è ad un pronome personale che viene associata l'azione verbale in caso di dislocazione del referente diretto: «il a dormi (, Pierre) / il a téléphoné (, Pierre) / il est arrivé (, Pierre)». 
scelta del $90 \%$ dei parlanti) e poi con (seconda scelta del $32 \%$ dei parlanti), con una percentuale di uso del $58 \%$ raggruppando le due varianti. La posizione iniziale del soggetto e, insieme, topic dell'enunciato costituisce il criterio di base per la costruzione della risposta. E questo si verifica con la classifica in terza posizione (o seconda, se si considera $\mathrm{S}(,) \mathrm{V}$ come una possibilità unica) del c'è presentativo, che pone il soggetto nella proposizione principale (tra c'è e che) e rimanda il verbo in subordinata.

In compenso, per telefonare, è la variante VS a dominare l'insieme delle scelte possibili (35\%), senza, ed è questo che differenzia telefonare da arrivare, distaccarsi molto dalla sua opposta SV (24\%). A partire dai risultati che riguardano le tre prime posizioni e gli esclusi (cf. sintesi qua sotto), si vede chiaramente che la struttura VS deve principalmente la sua alta percentuale al fatto che costituisce la prima scelta del $64 \%$ dei parlanti e che ha una percentuale debole di esclusione (11\%). Invece, la struttura SV, esclusa al $27 \%$ e considerata canonica al $26 \%$, deve la sua alta percentuale più alla sua classifica tra le prime posizioni che al fatto che sia stata scelta come la risposta più naturale.

$\begin{array}{cccccc} & \text { Prima posizione } & \text { Seconda posizione } & \text { Terza posizione } & \ldots & \text { Escluso } \\ \text { VS } & 64 \% & 16 \% & 6 \% & & 11 \% \\ \text { SV } & 26 \% & 23 \% & 12 \% & \ldots & 27 \%\end{array}$

Infine, per quanto riguarda arrivare, verbo inaccusativo al quale è tradizionalmente associata la posizione postverbale del soggetto, la situazione è inversa a quella di dormire ed i parlanti considerano effettivamente l'enunciato VS come canonico $(82 \%$ dei parlanti contro solo $11 \%$ per la variante SV). Nel caso di arrivare, i dati sono più netti che per telefonare, verbo al quale attribuiamo uno statuto intermedio, ma non sono l'inverso di quelli che riguardano l'inergativo dormire.

A prescindere dal bisogno comunicativo di introdurre per la prima volta nell'universo del discorso una relazione argomento-predicato, come in risposta a «che cosa è successo?», la struttura effettiva dell'enunciato che risponde a questo bisogno comunicativo sembra dipendere, come mostrano i risultati del test, dalle caratteristiche del verbo presente.

Consideriamo prima quello che condividono dormire, telefonare e arrivare. A partire del contesto proposto e delle sei proposte, l'enunciato SV sarebbe utilizzato in quasi il $30 \%$ dei casi e il suo opposto VS nel $28 \%$ dei casi. ${ }^{18}$ Queste medie, ottenute a partire dai dati dei tre verbi, sono le uniche a superare il $20 \%$, e delle due strutture è SV che i parlanti rifiutano di meno per costruire un enunciato tetico.

\footnotetext{
18 Per gli altri enunciati, la media è inferiore al 20\%: quella di S,V è a un po' più del 16\%, quella di V,S è dell' 11\%, quella del c'è presentativo è quasi del 10\% e infine, quella della frase scissa è a un po' più del al $5 \%$.
} 
Invece, due tendenze opposte si osservano con dormire da un lato e telefonare-arrivare dall'altro lato, al livello della classifica globale dei diversi enunciati, identica per i due ultimi verbi, e per quanto riguarda la scelta dell'enunciato canonico. In effetti, per il primo verbo, adinamico e atelico, il soggetto è posto come base e poi si introduce la relazione soggetto-verbo $(\mathrm{S}(,) \mathrm{V})$. Il verbo viene marcato dalla sintassi e dalla prosodia come l'elemento con «il grado massimale di dinamismo comunicativo» (Sonicola 1981). Per i due altri verbi, dinamici, è piuttosto l'azione ad essere posta come base e lo scopo dell'asserzione diventa l'attribuzione di questa azione ad una persona in particolare (VS).

Di fatto, per la formazione di un enunciato tetico, la sintassi italiana preferisce focalizzare il soggetto quando è attivo ed agente di un'azione dinamica e focalizzare il verbo nel caso inverso (soggetto semi-agentivo e azione adinamica).

\subsubsection{Secondo contesto: «Sono andata a fare spese. E Maria?»}

Passiamo ora al secondo contesto, caratterizzato da almeno i seguenti tratti:

- La domanda sottintende che l'identificazione di Maria è acquisita dall'interlocutore e che lei ha fatto qualcosa in particolare durante l'assenza del locutore. In modo concreto, l'elemento soggetto è quindi già introdotto nella domanda di partenza.

- La richiesta di informazione verte sull'azione esatta che è associabile a Maria.

- Il locutore A suppone che B conosca la risposta, cioè che questo ultimo sappia quello che lei ha effettivamente fatto in quel preciso momento.

- Lo scopo della presa di parola di B è quindi introdurre l'azione legata alla persona evocata, usando ogni volta nella sua risposta uno dei tre verbi studiati.

$\mathrm{Al}$ contrario del contesto precedente, il referente del soggetto è qui presente fin dalla domanda. L'introduzione e l'identificazione di Maria precedono l'intervento di B, che deve allora stabilire un legame tra questa persona e l'azione che ha effettuato e sulla quale porta l'attenzione di A. Qui è il soggetto che costituisce il punto di partenza dell'asserzione ed è l'azione che le viene associata che importa al livello comunicativo e corrisponde al punto di arrivo dello scambio. Di conseguenza, visto che l'italiano è una lingua a soggetto nullo, il verbo è l'unico elemento necessario e sufficiente perché l'asserzione soddisfi la richiesta d'informazione. Il soggetto può in effetti essere espresso solo nella desinenza verbale senza essere rappresentato in modo esplicito nell'enunciato con la ripresa del SN o con un pronome personale. Sempre per i nostri tre verbi, vediamo come gli enunciati italiani tendAno a veicolare un altro tipo di informazione: l'argomento costituisce il tema della conversazione ed il predicato l'obiettivo della presa di parola.

${ }^{17}$ In una lingua a soggetto non nullo come il francese, è ad un pronome personale che viene associata l'azione verbale in caso di dislocazione del referente diretto: «il a dormi (, Pierre) / il a téléphoné (, Pierre) / il est arrivé (, Pierre)». 


\section{A) Dormire}

A: "Sono andata a fare spese. E Maria?"

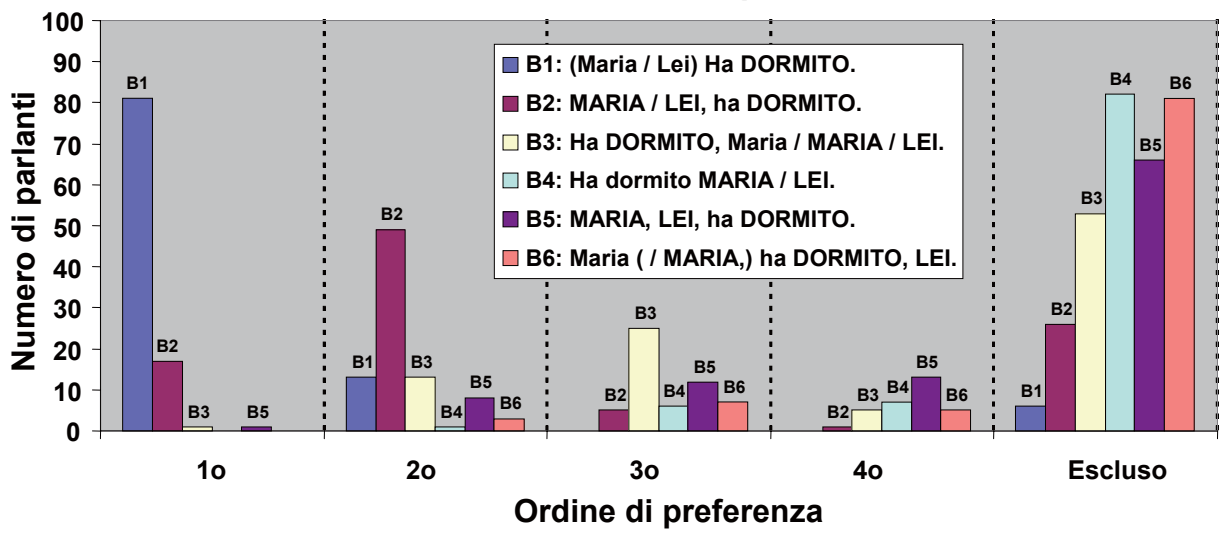

Totale dei punti per enunciato (percentuale)

A:' Sono andata a fare spese. E Maria?'

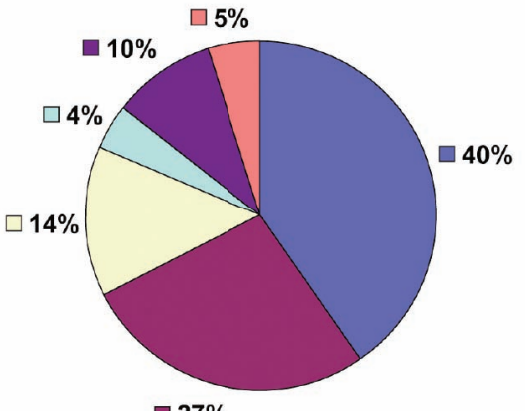

口B1: (Maria / Lei) Ha DORMITO.

口B2: MARIA / LEI, ha DORMITO.

口B3: Ha DORMITO, Maria / MARIA / LEI.

口B4: Ha dormito MARIA / LEI.

口B5: MARIA, LEI, ha DORMITO.

口B6: Maria ( / MARIA,) ha DORMITO, LEI.

Per il verbo dormire, i dati conducono alla gerarchizzazione seguente degli enunciati:

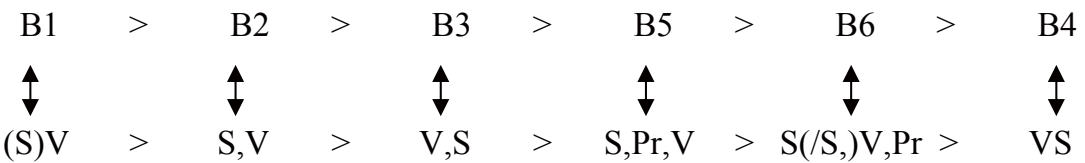

Vedremo in seguito che il contesto impone le stesse regole qualunque sia il verbo: la classifica è identica per i tre ${ }^{19}$ ed è solo in termini di proporzione che i risultati si distingono.

19 In tutti i casi, gli stessi quattro enunciati sono considerati come accettabili e i due altri rappresentano solo circa il 5\% dell'uso globale dell'insieme degli enunciati proposti. 
Come previsto nella descrizione delle caratteristiche del contesto imposto, è nel limitarsi a sottintendere il soggetto ed a asserire l'azione verbale (B1) ${ }^{20}$ che il parlante risponde alla domanda nel modo più pertinente. Scelto dall' $81 \%$ come enunciato canonico in questo contesto (cf. istogramma), (S)V domina di molto l'insieme delle altre strutture proposte.

Solo le dislocazioni a sinistra ed a destra, se vengono considerate come una struttura unica a due realizzazioni, hanno un peso rispetto all'enunciato dominante (con una percentuale totale d'uso di $41 \%$ rispetto all'insieme degli enunciati proposti). Tuttavia, anche se entrambe sono da considerare come adatte contestualmente, la scelta dei parlanti si orienta più facilmente verso la dislocazione a sinistra (27\%) che verso la dislocazione a destra (14\%). Il fatto che la metà dei parlanti classifichi seconda (dietro a (S)V) la dislocazione a sinistra mostra che, se il parlante valuta necessario riprendere il soggetto, lo metterà all'inizio dell'enunciato, come legame tra la domanda e la risposta ma anche come base dell'asserzione che lo riguarda.

Nel contesto imposto, nel quale solo il verbo veicola il contributo informativo, il soggetto è quindi di preferenza indicato dalla desinenza o dislocato. Sarà, al limite, rinforzato con un pronome tonico posto in inciso tra il soggetto e il verbo (la variante $\mathrm{S}, \mathrm{Pr}, \mathrm{V}$ rappresenta $10 \%$ dell'uso globale dei sei enunciati proposti) ma non occuperà la posizione focale, in finale e non dislocata (VS è escluso dal $82 \%$ dei parlanti).

\section{B) Telefonare}

\section{A: "Sono andata a fare spese. E Maria?"}

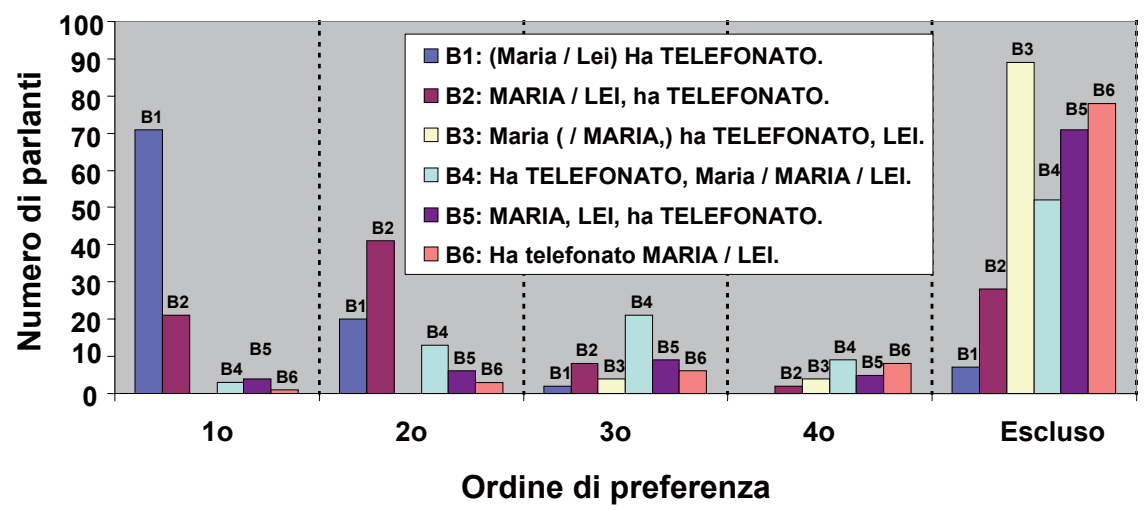

${ }^{20}$ L'unica proposta d'enunciato aggiunta da un parlante è identica a B1 al livello strutturale cambiando solo il tempo del verbo: «(Maria/Lei) Dormiva». Come ha suggerito giustamente Cristina Lavinio, con verbi come dormire (o come telefonare), al contrario di arrivare, l'imperfetto sembra comunque essere più normale e naturale in questo contesto, avendo la proprietà di insistere sulla durata, aspetto già presente nella loro semantica. 


\section{Totale dei punti per enunciato (percentuale)}

\section{A: "Sono andata a fare spese. E Maria?"}
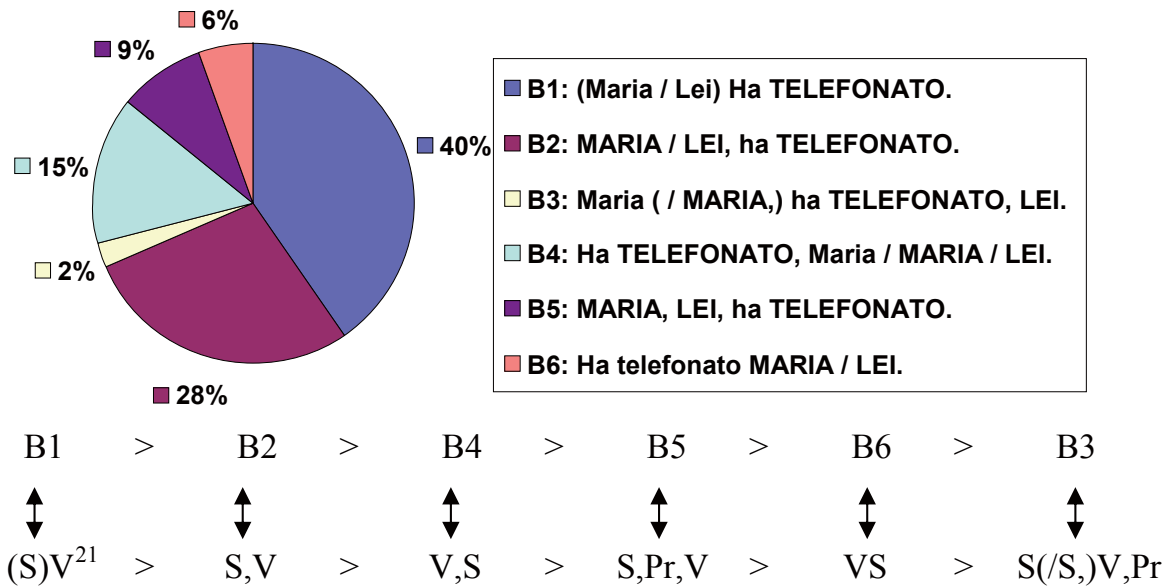

La scelta dei locutori ricade naturalmente sugli stessi enunciati per dormire e per telefonare. La classificazione differisce solo per le due varianti più rifiutate: $\mathrm{S}(/ \mathrm{S}$,)V,Pr precede VS con il primo verbo ( $5 \%$ contro $4 \%$ ) e lo segue con il secondo ( $2 \%$ contro $6 \%$ ). ${ }^{22}$ $\mathrm{E}$ anche per quanto riguarda le proporzioni, i risultati sono identici per il $3 \%$ circa: le esigenze legate al contesto agiscono aldilà di quelle legate al verbo, contrariamente a ciò che abbiamo potuto osservare nel caso precedente, cioè quando l'insieme SoggettoVerbo costituisce l'inizio della presa di parola.

\section{C) Arrivare}

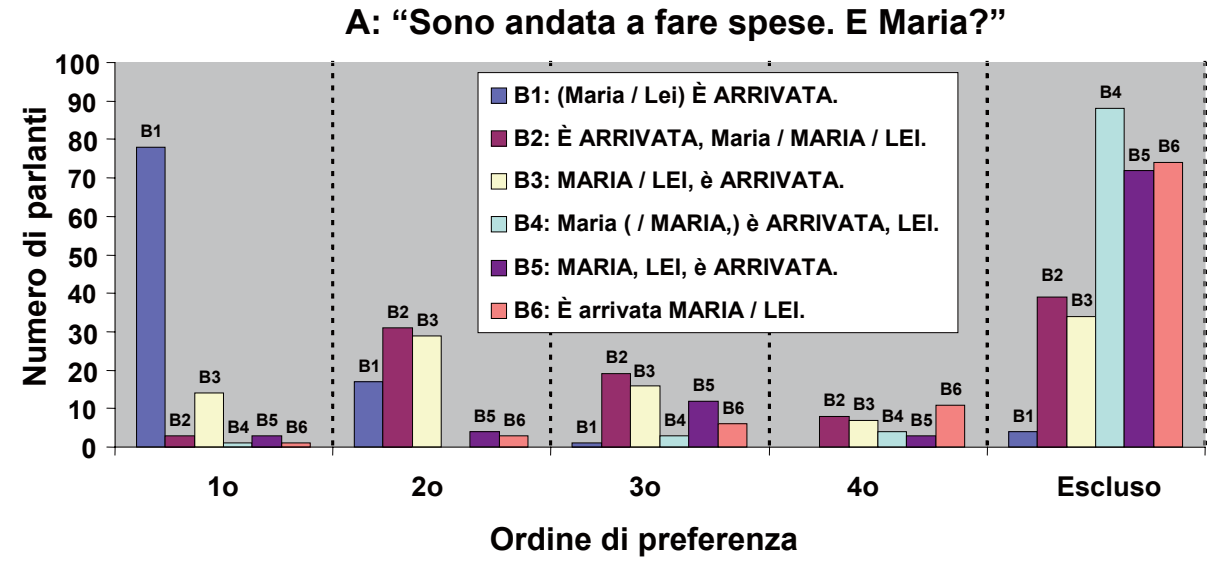

\footnotetext{
${ }^{21}$ Un parlante ci ha proposto come risposta «è stata al telefono»: telefonare viene sostituito da essere al telefono e il soggetto è solo marcato nella morfologia del verbo. Come con l'imperfetto, si sottolinea qua l'aspetto durativo dell'azione.

22 Osserviamo la stessa gerarchizzazione con il terzo verbo: $3 \%$ contro $8 \%$.
} 


\section{Totale dei punti per enunciato (percentuale)}

\section{A: "Sono andata a fare spese. E Maria?"}

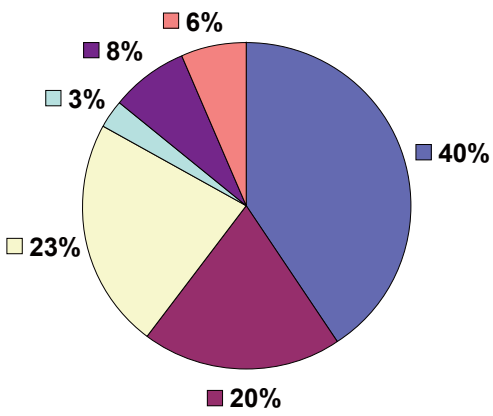

$\square$ B1: (Maria / Lei) È ARRIVATA.

口 B2: È ARRIVATA, Maria / MARIA / LEI.

$\square$ B3: MARIA / LEI, è ARRIVATA.

B4: Maria ( / MARIA,) è ARRIVATA, LEI.

B5: MARIA, LEI, è ARRIVATA.

$\square$ B6: È arrivata MARIA / LEI.

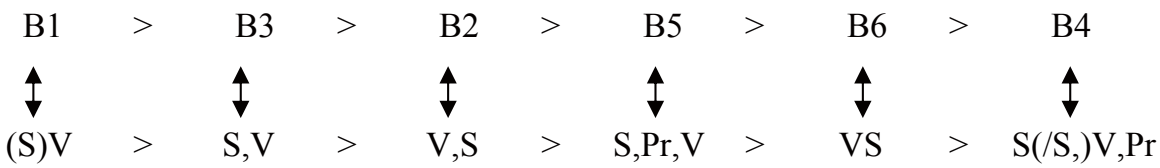

$\mathrm{Nel}$ caso di arrivare, come in quello dei verbi precedenti, i risultati sono molto simili in termini di classifica: la gerarchizzazione dei diversi enunciati è strettamente identica a quella osservata per telefonare e si distingue a quella che riguarda dormire solo per le strutture VS e S(/S,)V,Pr.

Invece, ed è questo punto che rende interessante il caso di arrivare, lo scarto tra i due tipi di dislocazione è minimo, al contrario di ciò che abbiamo visto per gli altri due predicati. In effetti, con dormire e telefonare, la dislocazione a sinistra precede la dislocazione a destra del $13 \%$, solo il $3 \%$ separa queste stesse strutture quando si tratta dell'ultimo verbo considerato ( $23 \%$ per $\mathrm{S}, \mathrm{V}$ contro $20 \%$ per $\mathrm{V}, \mathrm{S})$.

Come spiegare questa differenza notevole?

E perchè la dislocazione a destra si rivela adatta al contesto in modo quasi identico alla dislocazione a sinistra con arrivare?

Per quel che riguarda la prima domanda (lo scarto tra $\mathrm{S}, \mathrm{V}$ e V,S), i risultati parlano da sè: se il verbo considerato è arrivare, $\mathrm{S}, \mathrm{V}$ perde il $4 \%$ o $5 \%$ in termini di uso globale rispetto agli altri due verbi a vantaggio di V,S (più $6 \%$ rispetto a dormire $\mathrm{e}$ più $5 \%$ rispetto a telefonare). E anche se si considera solo la seconda scelta dei parlanti, questo rovesciamento di situazione salta agli occhi: con i due primi verbi, è la variante $\mathrm{S}, \mathrm{V}$ che occupa maggiormente il secondo posto (l'hanno classificata seconda 49 parlanti con dormire e 41 con telefonare); è invece la struttura V,S a sostituirla quando il verbo è arrivare, grazie alle 31 persone interrogate che hanno fatto questa scelta.

Perchè, in questo ultimo contesto, lo spostamento del soggetto alla destra di arrivare viene considerato come tanto naturale (dal 3\% circa) quanto il suo spostamento a sinistra? La struttura richiesta fin là da questo verbo, VS, è inadatta per rispondere 
in modo pertinente al bisogno comunicativo del parlante (esclusa da 74 parlanti su cento). Ma l'esigenza di posporre il soggetto al verbo è talmente forte per arrivare che tra i due tipi di dislocazione, la scelta va naturalmente sullo spostamento a destra dell'unico argomento e quindi sulla sua apparizione dopo il verbo nell'enunciato. Tra i tre verbi che abbiamo scelto di studiare, arrivare è l'unico che favorisce la posposizione del soggetto anche nella scelta del tipo di dislocazione: nell'impossibilità di poter associare l'enunciato VS al contesto proposto (il soggetto non essendo il focus), è $\mathrm{V}, \mathrm{S}$ che viene favorito.

Rispetto ai due contesti che abbiamo considerati in questo studio, telefonare si distingue da arrivare solo nel secondo, comportandosi piuttosto in maniera simile a dormire. Infatti, telefonare è un caso intermedio: tende da se stesso a posporre il soggetto quando è focale (come arrivare) ma non quando questo elemento è topicale (come dormire).

\section{D) Tabella riassuntiva e conclusioni}

Come abbiamo fatto finora, sintetizziamo i dati legati a questo secondo bisogno comunicativo: uno mi chiede che cosa ha fatto Maria, conosco la risposta ed affermo che questa persona ha dormito, ha telefonato o è arrivata. Ecco i dati per le due prime scelte:

$\begin{array}{lll}\text { Verbi } & \text { Prima posizione } & \text { Seconda posizione } \\ \text { DORMIRE } & 81 \% \text { per SV } & 49 \% \text { per } \mathrm{S}, \mathrm{V} \\ \text { TELEFONARE } & 71 \% \text { per VS } & 41 \% \text { per SV } \\ \text { ARRIVARE } & 78 \% \text { per VS } & 31 \% \text { per SV }\end{array}$

In termini di uso globale, a partire dal modo in cui è stato classificato ognuno degli enunciati dai parlanti, presentiamo ora le percentuali ottenute dalle sei sequenze proposte in rispondenza dei singoli verbi:

\begin{tabular}{|c|c|c|c|}
\hline Enunciati-risposte & DORMIRE & TELEFONARE & ARRIVARE \\
\hline$(\mathrm{S}) \mathrm{V}$ & $40 \%$ & $40 \%$ & $40 \%$ \\
\hline $\mathrm{VS}$ & $4 \%$ & $6 \%$ & $6 \%$ \\
\hline $\mathrm{S}, \mathrm{V}$ & $27 \%$ & $28 \%$ & $23 \%$ \\
\hline $\mathrm{V}, \mathrm{S}$ & $14 \%$ & $15 \%$ & $20 \%$ \\
\hline $\mathrm{S}, \mathrm{Pr}, \mathrm{V}$ & $10 \%$ & $9 \%$ & $8 \%$ \\
\hline $\mathrm{S}(/ \mathrm{S}, \mathrm{V}, \mathrm{Pr}$ & $5 \%$ & $2 \%$ & $3 \%$ \\
\hline
\end{tabular}

(In grassetto la percentuale più alta per ogni verbo.)

A partire dal contesto, che impone che il verbo sia focale ed il soggetto topicale nell'enunciato-risposta, e visto che l'italiano è una lingua a soggetto nullo, una sola ed 
unica struttura risulta totalmente adatta qualunque sia il verbo presente: un soggetto necessariamente marcato nella desinenza verbale, accessoriamente ripetuto all'inizio, ed un verbo in posizione finale e che riceve l'accentuazione principale.

Nello stesso modo per i tre verbi, la variante opposta, VS, viene considerata come inadeguata al contesto. ${ }^{23}$ L'esclusione di quest'ordine sembra dovuta solo a fattori comunicativi: l'ordine VS viene associato alla focalizzazione del soggetto (il soggetto veicola «un'informazione nuova» nel 75\% degli ordini VS del corpus di Sornicola 1994: 47). Invece, il contesto impone al verbo di costituire da solo l'informazione da trasmettere e di conseguenza il soggetto non può occupare la posizione finale, riservata agli elementi focalizzati.

Lo stesso avviene alla presenza in finale di un pronome tonico di rinforzo $(\mathrm{S} / \mathrm{S},) \mathrm{V}, \mathrm{Pr}) .{ }^{24}$ Solo quando il pronome tonico viene posto direttamente dopo il soggetto, che ne risulta così rafforzato, i parlanti accettano la presenza di questo elemento (tacco di esclusione inferiore o uguale a $72 \%$ per i tre verbi).

I risultati sono diversi relativamente al verbo, solo per quanto riguarda le dislocazioni, che occupano le seconde $(\mathrm{S}, \mathrm{V})$ e terze $(\mathrm{V}, \mathrm{S})$ posizioni della classifica. Se per i primi due verbi, la scelta va largamente sulla dislocazione a sinistra del soggetto, per il terzo, la differenza di pertinenza tra le due strutture è molto meno ovvia. Sembra che l'influenza del verbo arrivare sulla posizione del suo argomento unico sia abbastanza forte per condurre il parlante italiano a considerare la dislocazione a destra del soggetto tanto pertinente quanto la sua dislocazione a sinistra. Bisognerebbe allora studiare quello che succede quando, per esempio, il verbo è focus di tipo contrastivo e il soggetto topico (A: «Piero è partito?» B: «No. Arrivare + Piero») o quando l'enunciato è semplicemente la conferma di una supposizione precedente (A: «Piero è (ben) arrivato?» B: «Si. Arrivare + Piero»), per poter verificare o invalidare l'ipotesi che la preferenza per la dislocazione a destra sia direttamente legata alle esigenze strutturali di questo verbo.

\section{Conclusione generale}

La ricerca che abbiamo proposto in questo articolo voleva porre a confronto tre verbi con struttura argomentale identica ma con proprietà semantiche ed aspettuali distinte, in due contesti comunicativi possibili, mediante uno studio realizzato su un campione di cento parlanti nativi.

Tra i verbi considerati, c'era prima dormire, che richiede un solo argomento, il soggetto («qualcuno dorme»), che rinvia necessariamente ad un essere animato il cui stato di agente è abbastanza debole. Il secondo era telefonare, considerato come un verbo monoargomentale costruito con un argomento animato e agentivo, anche

${ }^{23}$ L'enunciato è escluso dal $82 \%$ per dormire, dal $78 \%$ per telefonare e dal $74 \%$ per arrivare.

24 Questa variante è rifiutata dall' $81 \%$ quando il verbo è dormire, dall' $89 \%$ quando si tratta di telefonare e dall' $88 \%$ se il verbo è arrivare. 
se ha la possibilità di avere un paziente («qualcuno telefona (a qualcuno)»), che può rimanere sottinteso. L'ultimo, arrivare, richiede invece un argomento soggetto agentivo, se è umano (e non è sempre il caso), e suppone un altro dato: il luogo («qualcuno arriva (da qualche parte)»). Questi tre verbi hanno comunque esplicitamente bisogno solo di un argomento soggetto per essere costruiti. Al livello dell'ausiliare, i primi due verbi hanno bisogno di avere, l'ultimo di essere. In termini di aspetto, abbiamo considerato dormire come un verbo adinamico ed atelico, telefonare come un verbo dinamico ed atelico ed arrivare come un verbo dinamico e telico.

Una volta identificate le caratteristiche di ognuno dei tre verbi, ci siamo dedicati alle diverse realizzazioni di una stessa struttura, contenente solo il predicato e l'argomento soggetto, in due situazioni di comunicazione precise. Nel primo contesto imposto, il soggetto ed il verbo avevano lo stesso peso comunicativo e la loro associazione costituiva l'apporto informativo; nel secondo contesto, solo il verbo veicolava l'informazione pertinente, questa richiesta dall'interlocutore.

Prima di esporre le conclusioni generali della ricerca, vogliamo semplicemente ribadire il fatto che, a partire dal momento in cui una struttura argomentale viene «trasformata» in enunciato, due tipi di influenza entrano in gioco: quella direttamente legata agli elementi combinati e quella legata al contesto (cf. schema qua sotto). Le richieste possono andare nello stesso senso o essere in contraddizione: da qui l'interesse a metterle a confronto per poter rendere conto della struttura effettiva degli enunciati:

\section{Predicato + Argomento $\longleftrightarrow$ ENUNCIATO $\longrightarrow$ Contesto}

Nel primo contesto, nel quale si trattava di introdurre la relazione argomento-predicato, senza che né l'uno né l'altro di questi elementi fossero stati già introdotti, siamo arrivati alla conclusione che la struttura considerata più adeguata a questo bisogno non è dipendente dal contesto ma dal verbo e dall'argomento presenti. Perché? Semplicemente perché i risultati che riguardano l'enunciato canonico variano in modo molto significativo se il verbo è dormire e se il verbo è telefonare o arrivare.

Che il contesto abbia un'influenza, è innegabile: rigetta le strutture inadeguate ed ammette o favorisce le altre ma non ne impone una come canonica. In effetti, sono le caratteristiche situazionali dello scambio che si oppongono alla pertinenza della frase scissa, che ammettono come possibili le dislocazioni del soggetto ed il c'è presentativo e che attribuiscono il massimo dell'adeguatezza alle strutture SV e VS. I risultati sono omogenei qualunque sia il verbo per le diverse proposizioni, tranne che per i due enunciati classificati migliori: il contesto non è sufficiente per isolare la costruzione più adatta e sono gli elementi presenti ad imporre l'una $o$ l'altra come canonica.

Per formare un enunciato tetico, l'italiano attribuisce al verbo la posizione focale, cioè in finale ed associata all'accentazione principale quando l'azione è adinamica, atelica e il soggetto è solo semi-agentivo, come è il caso di dormire e del suo argomento unico. Invece, questa stessa posizione viene riservata al soggetto quando quest'ul- 
timo governa un'azione verbale dinamica, come con telefonare, arrivare e i loro soggetti. L'enunciato tetico ottenuto introduce direttamente la relazione soggetto-verbo (SV) quando l'aspetto dinamico dell'uno come dell'altro dei due elementi non è evidente e focalizza il soggetto (VS) quando il suo referente è alla base della realizzazione dell'azione, cioè quando lo scopo dell'asserzione è non solo di associare un referente ed un'azione ma soprattutto di attribuire l'azione ad una persona particolare, che ne è il punto di partenza.

Il secondo contesto, invece, attribuiva uno statuto comunicativo diverso ai due elementi: il verbo veicolava l'informazione richiesta dall'interlocutore, il soggetto invece era (solo) il tema dell'interazione.

Cominceremo ricordando ancora una volta che in italiano non è necessaria la presenza «esplicita» del soggetto: la sua espressione può limitarsi alle marche verbali (tranne se sono ambigue). Da questa opzionalità deriva che la scelta tra presenza o assenza di questo costituente viene direttamente legata al valore comunicativo che gli è attribuito nell'enunciato. Nel nostro contesto, l'argomento unico è presupposto e viene affermato solo il predicato. La presenza esplicita del soggetto è considerata dai parlanti nativi come accessoria, il verbo invece e l'azione alla quale rinvia costituiscono lo scopo dell'intervento. È quindi questo secondo costituente, la cui presenza è richiesta dal contesto, che riceve tutte le marche della prominenza nell'enunciato-risposta (può comparire solo, occupare la posizione finale se il soggetto è espresso di nuovo, non è distaccato, riceve l'accentazione principale...).

Se la presenza del soggetto in posizione iniziale (SV) è contestualmente ammessa, la realizzazione di questo stesso argomento in posizione finale (VS) è rifiutata e l'enunciato deve essere considerato come inadeguato. Ed è lo stesso per le due proposizioni di risposta che contengono un pronome tonico che rinforza il soggetto. La conclusione da trarre da questi risultati è che il contesto qui esclude tutte le strategie che nell'enunciato contribuiscono ad attribuire lo statuto di elemento focale al soggetto. Tuttavia, il fatto che l'argomento non possa ricevere le marche proprie al focus non gli toglie la possibilità di essere considerato come un elemento prominente nel discorso: non lo è quando viene solo espresso attraverso la morfologia del verbo o quando è distaccato a destra, in appendice, ma la volontà di sottolineare la sua funzione tematica nell'enunciato è evidente quando è posto all'inizio e ancora di più quando è dislocato a sinistra.

Il contesto tiene a questa prominenza attribuita al soggetto, elemento la cui presenza, ricordiamo, è facoltativa:

$$
\text { 9. } \mathrm{V}>\mathrm{SV}>\mathrm{S}, \mathrm{V}>\mathrm{V}, \mathrm{S} \quad \longrightarrow \quad(\mathrm{S}(,)) \mathrm{V}>\mathrm{V}, \mathrm{S}
$$

In modo globale, il distacco in posizione iniziale del soggetto $(S, V)$ è in effetti considerato dai parlanti come più adatto al contesto che la sua realizzazione identica in finale $(\mathrm{V}, \mathrm{S})$. Per dormire e telefonare, la prima struttura domina largamente poiché, in termini di percentuale di uso, più del 10\% la separano della seconda. Invece, le esigenze legate al verbo arrivare sono abbastanza forti per limitare lo scarto al solo $3 \%$. Infatti, l'influenza che ha il verbo sotto la posizione del suo argomento è debole 
rispetto all'inadeguatezza nel contesto di VS (il soggetto non essendo focus qua) ma sembra essere in misura tale da condurre il parlante italiano a considerare in questo caso la posizione del soggetto a destra $(\mathrm{V}, \mathrm{S})$ altrettanto pertinente quanto la sua posizione a sinistra $(\mathrm{S}, \mathrm{V})$. Tuttavia, come abbiamo detto prima, bisognerebbe considerare altri contesti per confermare o smentire questa ipotesi, secondo la quale sarebbe dal verbo e dalle sue esigenze strutturali che proverrebbero la pertinenza della posizione a destra in un contesto dato.

In modo più generale, il lavoro che abbiamo appena esposto ci ha permesso di arrivare alle conclusioni e osservazioni seguenti:

- una stessa struttura argomentale non conduce necessariamente ad una realizzazione identica degli elementi al livello enunciativo, cioè una struttura predicato(+argomento) per esempio, come è il caso per dormire, telefonare ed arrivare, non conduce sempre agli stessi enunciati canonici in un contesto identico. La struttura argomentale permette semplicemente l'identificazione degli elementi necessari e dipendenti del predicato ma non determina la forma dell'enunciato.

- Il contesto fornisce delle «leggi generali», che valgono per ogni verbo e per ogni argomento: rigetta le marche inadatte (un soggetto focus non sarà distaccato a destra e disaccentuato per esempio), ammette quelle che permettono una buona interpretazione del bisogno comunicativo (un elemento tematico può essere introdotto di nuovo per maggiore chiarezza) e favorisce le strutture più pertinenti da un punto di vista qualitativo (trasparenza dell'enunciato) e quantitativo (economia).

- Quando il predicato rinvia ad un'azione dinamica e telica e/o ha un argomento unico agentivo, la posizione focale è riservata a questo argomento, a meno che lo scopo dell'intervento sia solo quello di introdurre la parte verbale. E invece, se l'azione è adinamica, atelica e l'argomento solo semi-agentivo, la posizione focale è riservata al verbo, tranne quando il bisogno comunicativo riguardi solo il suo soggetto.

- La forma effettiva di un enunciato adeguato contestualmente non è solo legata alla situazione comunicativa nella quale viene inserita. È piuttosto direttamente dipendente dal contesto in primis, e secondariamente influenzata dalle proprietà semantiche ed aspettuali degli elementi presenti nell'enunciato (come abbiamo visto per S,V e V,S con arrivare nel secondo contesto). Le esigenze contestuali dominano quelle relative ai predicati e argomenti, che promuovono solo una certa strutturazione dell'enunciato senza imporla.

La scelta dell'italiano come oggetto di studio ci ha anche permesso di analizzare il peso nel contesto, della possibilità di inversione (libera) del soggetto e della presenza «opzionale» di questo argomento.

Prima di tutto, abbiamo avuto la conferma che la pertinenza della postposizione del soggetto al verbo è direttamente dipendente dal contesto e quindi dallo statuto comunicativo del primo argomento nell'enunciato.

Poi, per quanto riguarda il fatto che la desinenza verbale possa permettere solo l'identificazione dell'elemento soggetto, due osservazioni s'impongono: la prima è che questa possibilità offerta dalla lingua è sfruttata al suo massimo appena è permessa contestualmente (cf. secondo contesto); la seconda è che se, malgrado questa pos- 
sibilità, la scelta del parlante è di esprimere esplicitamente il soggetto, quest'ultimo sarà marcato nell'enunciato come un elemento prominente. A partire dal momento in cui la presenza del primo argomento è accessoria rispetto al contesto, abbiamo riscontrato due tendenze radicalmente opposte: sia che gli si accordi un valore comunicativo molto debole, limitando la sua espressione alla morfologia del verbo o distaccandolo a destra, sia che si «sottolinei» nell'enunciato il suo statuto prominente di tema dello scambio, con la sua realizzazione «esplicita» in posizione iniziale (SV) o con la sua dislocazione a sinistra e l'accentuazione che gli viene associata $(\mathrm{S}, \mathrm{V})$.

In questo articolo, ci siamo limitati a considerare tre verbi e due contesti distinti, pur decisamente consapevoli che sarebbe opportuno allargare lo studio ad altri predicati mono-argomentali ed ad altri bisogni comunicativi per poter proporre un'analisi più completa della loro influenza rispettiva sulla forma dell'enunciato e, di conseguenza, per poter fornire una tipologia delle differenti strutture e del loro uso in maniera molto più pertinente.

\section{Bibliografia}

Albano Leoni, Federico/Eleonora Stenta Krosbakken/Rosanna Sornicola/Carolina Stromboli (a cura di), Dati empirici e teorie linguistiche. Roma: Bulzoni, 455-476.

Andorno, Cecilia (2003) La grammatica italiana. Milano: Paravia Bruno Mondadori Editori.

BENINCÀ, Paola (1994) La variazione sintattica. Bologna: il Mulino.

BERNINI, Giuliano (1995) «Verb-subject order in Italian: an investigation of short announcements and telecast news.» Sprachtypologie und Universalienforschung 48/1-2, 44-71.

Bonvino, Elisabetta (2005) Le sujet postverbal. Une étude sur l'italien parlé. Paris: Ophrys.

Burzio, Luigi (1986) Italian syntax. Dordrecht: D. Reidel Publishing Company.

Cennamo, Michela (2001) «L'inaccusitività in alcune varietà campane: teorie e dati a confronto.» In: F. Albano Leoni et al. (a cura di), 427-453.

Chol, Hye-Won (1999) Optimizing structure in context: scrambling and information structure. Stanford: CSLI publications.

CoRNISH, Francis (2001) «L'inversion 'locative' en français, italien et anglais: propriétés syntaxiques, sémantiques et discursives.» Cahiers de grammaire 26, 101-123.

Costabile, Norma (1967) Le strutture della lingua italiana: grammatica generativo-trasformativa. Bologna: Riccardo Patròn.

CRESTI Emanuela (1987) «L'articolazione dell'informazione nel parlato.» In: Gli italiani parlati: sondaggi sopra la lingua di oggi. Firenze: presso l'Accademia della Crusca, 27-90.

Frison, Lorenza (1988) «La struttura della frase semplice.» In: L. Renzi et al. (a cura di), Grande grammatica italiana di consultazione. Vol. I. Bologna: il Mulino, 47-87.

Genot, Gérard (1989) Système de la langue italienne. Nanterre: Erasme.

GrAFFI, Giorgio (1994) Le strutture del linguaggio. Sintassi. Bologna: il Mulino.

LOPORCARO, Michele (2001) «La selezione dell'ausiliare nei dialetti italiani: dati e teorie.» In: F. Albano et al. (a cura di), 455-476.

Marandin, Jean-Michel (2003) «Inversion du sujet et structure de l'information dans les langues romanes.» In: D. Godard (a cura di), Langues romanes. Problèmes de la phrase simple. Paris: Editions du CNRS, 345-392. 
MATRAS, Yaron/Hans-Jürgen SASSE (a cura di) (1995) Verb-subject order and theticity in European languages. Numero speciale di Sprachtypologie und Universalienforschung 48/1-2.

Milano, Emma (2005) «A proposito delle dislocazioni a sinistra: alcune osservazioni preliminari sulla variazione in un corpus di italiano parlato.» In: E. Burr (a cura di), Tradizione e innovazione, Il parlato: teoria - corpora - linguistica dei corpora. Firenze: Franco Cesati Editore, 371-381.

PINTO, Manuela (1997) Licensing and interpretation of inverted subjects in italian. Utrecht: LED. SASSE, Hans-Jürgen (1987) «The thetic/categorical distinction revisited.» Linguistics 25, 511-580.

SASSE, Hans-Jürgen (1995) "Theticity and VS ordrer: a case study.» Sprachtypologie und Universalienforschung 48/1-2, 3-31.

SoRnicola, Rosanna (1981) Sul parlato. Bologna: il Mulino.

SoRNICOLA, Rosanna (1994) «On word order variability: a study from a corpus of Italian.» Lingua e Stile 29/1, 25-57.

SoRNICOLA, Rosanna (1995) «Theticity, VS order and the interplay of syntax, semantics and pragmatics.» Sprachtypologie und Universalienforschung 48/1-2, 72-83.

TAMBURINI, Guido (1998) «L'ordine dei costituenti e l'articolazione dell'informazione in italiano: un'analisi distribuzionale.» Studi di grammatica italiana XVII, 399-443.

TESNiÈRE, Lucien (1959) Eléments de syntaxe structurale. Paris: Editions Klincksieck.

VALLDUVí, Enric (1992) The informational component. New York: Garland.

\section{Riassunto \\ SOGGETTO E VERBO CON DORMIRE, TELEFONARE E ARRIVARE IN CONTESTI DIVERSI}

L'articolo presenta i risultati di uno studio realizzato su un campione di cento parlanti attraverso la somministrazione di un questionario. Il suo scopo è di descrivere il legame esistente tra contesto verbale e forma dell'enunciato in italiano. Il questionario consiste nella presentazione di una serie di contesti linguistici, ai quali sono associati diversi tipi di frasi, chiedendo ai parlanti di esprimere un giudizio sull'adeguatezza di ogni variante rispetto al contesto proposto. L'articolo si limita a presentare la realizzazione della sequenza soggetto+verbo in due contesti diversi per i verbi dormire, telefonare e arrivare, distinti per proprietà semanticoaspettuali ma identici dal punto di vista della struttura argomentale. Infatti, questi verbi hanno un solo argomento: il soggetto, che può anche rimanere implicito, essendo sintatticamente opzionale in italiano; di conseguenza, tutti gli altri elementi che si aggiungano eventualmente all'enunciato, risultano indipendenti dal verbo. I due contesti linguistici messi a confronto si presentano in questo caso sotto forma di domande e determinano, nelle risposte, una organizzazione diversa dell'informazione: nel primo («che cosa è successo?») né il soggetto né il verbo sono presupposti e la loro introduzione è complessivamente informativa; nel secondo («sono andata a fare spese. E Maria?») solo il soggetto è introdotto ed è il verbo a portare l'informazione «nuova». Sulla base di queste restrizioni contestuali, lo scopo è di analizzare quali siano le strategie linguistiche adeguate o meno per veicolare il tipo di informazione richiesto, confrontando i risultati per i tre verbi, al fine di stabilire un legame tra proprietà degli elementi presenti, contesto e forma dell'enunciato. 


\section{Povzetek \\ OSEBEK IN GLAGOL Z DORMIRE, TELEFONARE IN ARRIVARE V RAZLIČNIH KONTEKSTIH}

Članek predstavlja rezultate raziskave, opravljene s pomočjo vprašalnika na vzorcu stotih govorcev. Namen članka je opisati zvezo med jezikovnim kontekstom in obliko izjave v italijanščini. V vprašalniku je podana vrsta jezikovnih kontekstov, s katerimi so povezani različni tipi stavkov, anketiranci pa so naprošeni, da se izrečejo o ustreznosti vsake posamezne različice glede na predlagani kontekst. Članek se omejuje na predstavitev zaporedja osebek + glagol v dveh različnih kontekstih z glagoli dormire, telefonare in arrivare, ki se med sabo razlikujejo po semantično-aspektualnih lastnostih, a so enaki z vidika strukture argumentov. Ti glagoli imajo namreč en sam argument, in sicer osebek, ki lahko ostane tudi impliciten, saj je v italijanščini skladenjsko neobvezen; posledično so vsi ostali elementi, ki bi se lahko dodali k izjavi, od glagola neodvisni. Med seboj primerjana jezikovna konteksta sta v našem primeru predstavljena v obliki vprašanj, v odgovorih pa izzoveta vsakič različno organizacijo podatkov: v prvem kontekstu (»che cosa è successo «) ne osebek ne glagol nista predpostavljena in tako je njuna pojavitev kot takšna informativna; v drugem (»sono andata a fare spese. E Maria?«) se pojavi samo osebek in tako je glagol tisti, ki prinaša »novo« informacijo. Upoštevajoč te kontekstualne omejitve, ima prispevek namen proučiti, katere jezikovne strategije uspešno oz. neuspešno prenašajo zahtevano vrsto informacij. Podana je primerjava rezultatov za izbrane tri glagole, da bi tako lahko ugotovili zvezo med lastnostmi danih elementov, kontekstom in obliko izjave. 